\title{
Seine Heiligkeit Benedikt XVI. - Doktor honoris causa der Päpstlichen Universität Johannes Paul II. in Krakau
}

Der Rektor der Päpstlichen Universität Johannes Paul II. in Krakau (UPJPII), H. H. Prof. Dr. hab. Wojciech Zyzak, und der Rektor der Akademie für Musik in Krakau (AM), Prof. Dr. hab. Zdzisław Łapiński, bereiteten gemeinsam einen Brief vor, den sie mit der Bitte an den emeritierten Papst Benedikt XVI. sandten, den beiden Hochschulen die Ehre zu erweisen, ihn mit dem Titel Doktor honoris causa auszeichnen zu dürfen. Diese Bemühungen wurden auch durch ein Schreiben von Kardinal Stanisław Dziwisz, dem Großkanzler der UPJPII wohlwollend unterstützt. Anfang Februar 2015 antwortete der emeritierte Papst schließlich, dass er ausnahmsweise von seinem Grundsatz abweichen wolle, nach seinem Rückzug in die Stille abseits der Öffentlichkeit keinerlei Auszeichnungen mehr anzunehmen: „Das Angebot, das mir die Akademie für Musik in Krakau sowie die Päpstliche Universität Johannes Paul II. in Krakau unterbreitet haben, stellt jedoch eine wahrhafte Auszeichnung dar. Ich bin mir bewusst, dass meine Texte zur Kirchenmusik allein diese Auszeichnung nicht verdienen. Aber die Freude darüber, auf diese Weise meinem verehrten und geliebten Vorgänger, dem heiligen Johannes Paul II., wieder nahe sein zu können, ist so groß, dass ich zu dieser Ehrung nicht NEIN sagen kann. Mit Dankbarkeit und Freude nehme ich daher das Doktorat honoris causa Ihrer geschätzten Institutionen an”.

Darauf hin begannen an beiden Hochschulen die erforderlichen Prozeduren, die mit der Verleihung der Ehrendoktorwürde an Seine Heiligkeit Benedikt XVI. verbunden waren. Am 20. April 2015 fasste der Senat der Päpstlichen Universität Johannes Paul II. in Krakau den Beschluss, den 
emeritierten Papst mit dem Titel eines Doktors honoris causa der UPJPII auszuzeichnen. Entsprechend der Vorgaben wurden zunächst positive Rezensionen vorbereitet: H. H. Prof. Dr. hab. Jacek Bramowski (AM), Prof. Dr. hab. Teresa Malecka und H. H. Prof. Dr. hab. Andrzej Zając. Zum Laudator erwählte man Prof. Dr. hab. Stanisław Krawczyński. H. H. Prof. Dr. hab. Jacek Bramowski unterstrich in seiner Rezension unter anderem: „Die Theologie von Benedikt XVI. weckt im modernen Menschen die Sehnsucht, von Neuem den tieferen Sinn seiner Existenz zu entdecken. Für ihn ist das nicht die eine oder andere Ideologie sondern allein der personale Gott, zu dem wir auch über den Weg des Schönen gelangen können, das in der Musik gegenwärtig ist. Der Heilige Vater Benedikt XVI. hat unserer Welt diese Hoffnung konsequent aufgezeigt und damit die Entscheidung der Päpstlichen Universität Johannes Paul II. in Krakau über die Verleihung der Ehrendoktorwürde an ihn umfassend begründet. Mit der Bitte an Seine Heiligkeit, diese Ehrung anzunehmen, drücken wir die demütige Huldigung seiner vielfältigen Reflexionen und seines segensreichen Wirkens aus". Prof. T. Malecka betonte dagegen in ihrer Rezension unter anderem, es sei vortrefflich, dass „die Päpstliche Universität Johannes Paul II. in Krakau sowie die Akademie für Musik in Krakau die Initiative ergriffen haben, einen großen Menschen, einen unermüdlichen Verkünder der höchsten Werte auch mit der höchsten akademischen Würde zu ehren und damit den Standard der universitären Kultur zu heben wie auch den Wert des akademischen Ethos zu bereichern. Mit dem Einverständnis des emeritierten Heiligen Vaters Joseph Ratzinger, das Doktorat honoris causa anzunehmen, erweist er unseren beiden akademischen Institutionen die höchste Ehre”. H. H. Prof. A. Zając erinnerte in seiner Rezension unter anderem daran, dass "die Verleihung des Ehrendoktortitels an den Heiligen Vater Benedikt XVI. durch zwei Hochschulen in Krakau, welche die Musik gemeinsam am universitätsübergreifenden Institut für Kirchenmusik lehren, besondere Symbolkraft besitzt... Auf diese Weise wollen die beiden Hochschulen dem Heiligen Vater ihre Ehrerbietung und die große Dankbarkeit für seine intensiven und außerordentlich wertvollen theologischen Reflexionen über die Musik ebenso Ausdruck verleihen wie auch dafür, dass er über viele Jahre hinweg in allen Ämtern, die er bekleidet hat, mit besonderer Sorge auf die edle Schönheit der Kirchenmusik und auf deren spezifischen Stellenwert in der heiligen Liturgie der Kirche Christi verwiesen hat".

Die Ehrendoktorwürde wurde Seiner Heiligkeit Benedikt XVI., dem emeritierten Papst (24. Doktor honoris causa der UPJPII), insbesondere zuerkannt: 


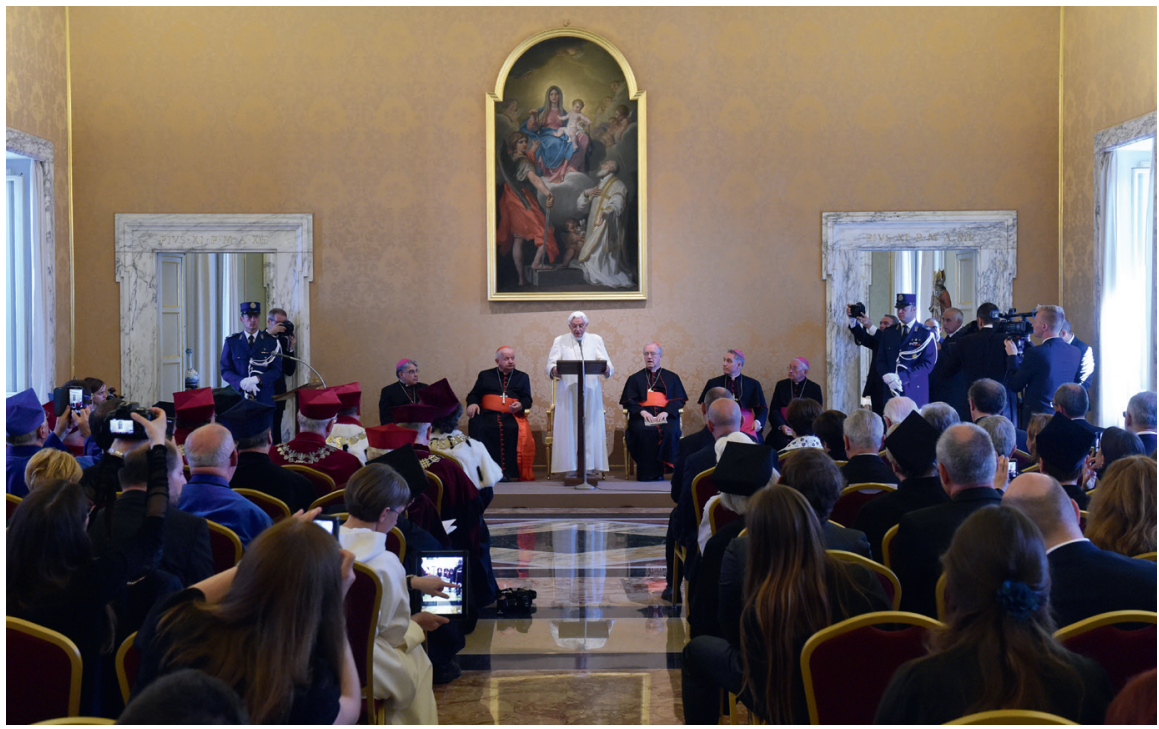

- für seine große Wertschätzung, die er der musikalischen Tradition der Kirche entgegen gebracht hat, und seine Sensibilität für den Dialog von Musik und Glauben;

- für seine unermüdliche Sorge um die edle Schönheit der Kirchenmusik und deren spezifischen Stellenwert in der heiligen Liturgie der Kirche, die er zeit seines Lebens in allen Ämtern stets zum Ausdruck gebracht hat;

- für die Betonung der Bedeutung der via pulchritudinis, die nach seiner Lehrmeinung auch für den modernen Menschen ein Weg sein kann, um Gott kennen zu lernen und ihm Ehre zu erweisen;

- für das mutige Zeugnis der Wahrheit, das er mit seinem Leben, seinem Dienst, seiner Lehre und seiner wissenschaftlichen Arbeit abgelegt hat, einer Wahrheit, die den christlichen Glauben in den verworrenen Zeiten von Liberalismus, Postmodernismus und Relativismus gestärkt und Europa die Rückbesinnung auf seine geistige Dimension nahegebracht hat;

- für seine wohlwollende Unterstützung bei der Umgestaltung der Päpstlichen Theologischen Akademie hin zur Päpstlichen Universität Johannes Paul II. in Krakau.

Am 4. Juli 2015 wurde nun die Reihe der Ehrendoktoren der Päpstlichen Universität Johannes Paul II. sowie der Akademie für Musik in Krakau durch eine außerordentlich prominente und vortreffliche Persönlichkeit erweitert - 
Seine Heiligkeit Benedikt XVI. Einen Tag vor der Feierlichkeit, am 3. Juli 2015, versammelten sich Professoren, Studenten und weitere Teilnehmer zu einer hl. Messe im Petersdom. Gemeinsam mit dem Hauptzelebranten H. H. Kardinal Stanisław Dziwisz beteten sie am Grab des hl. Johannes Paul II. für die Anliegen der Erzdiözese Krakau, der Päpstlichen Universität Johannes Paul II. und der Akademie für Musik in Krakau. Die feierliche Verleihung des Ehrendoktortitels der UPJII und der AM in Krakau fand um 10 Uhr in Castel Gandolfo statt. Teilnehmer waren Professoren und Studenten beider Hochschulen, geladene Gäste sowie Freunde des Geehrten und des früheren Papstes Johannes Pauls II. Die musikalische Umrahmung übernahmen der Chor „Psalmodia” der UPJII und das Streichquartett „Ypsylon” der Akademie für Musik. In seiner Begrüßung wandte sich Kardinal St. Dziwisz, Metropolit von Krakau und Großkanzler der UPJII, mit folgenden Worten an den emeritierten Papst: „Heiliger Vater! Vor allem möchte ich ganz besonders unsere unermessliche Freude über diese heutige, wahrlich nicht alltägliche Begegnung ausdrücken... Die Päpstliche Universität Johannes Paul II., die von Ihrer Heiligkeit am 19. Juni 2009 bestellt wurde, sowie die Akademie für Musik in Krakau haben beschlossen, unserer Dankbarkeit gemeinsam Ausdruck zu verleihen und dem Heiligen Vater mit dem Doktorat honoris causa zu ehren. Dass Sie diese Auszeichnung ausnahmsweise annehmen, werten wir als Hochachtung Ihrer Heiligkeit für den heiligen Johannes Paul II. Er selbst - der heilige Papst - war stets von Dankbarkeit für seinen allernächsten Mitarbeiter - den Präfekten der Glaubenskongregation erfüllt. Wir sind sicher, dass er jetzt von oben auf uns blickt und sich über unsere Begegnung freut. Die Rektoren der beiden Krakauer Hochschulen werden im weiteren Verlauf noch genauer über die Begründungen sprechen, die zu dem Beschluss ihrer Institutionen führten, Ihrer Heiligkeit den Ehrendoktortitel $\mathrm{zu}$ verleihen. Ich möchte nur hinzufügen, dass der Name des Heiligen Vaters Benedikt XVI. für immer in die Geschichte der Päpstlichen Universität Johannes Paul II. in Krakau eingeschrieben sein wird. Wir lieben Sie, Heiliger Vater, und werden Sie ganz sicher immer in unser Gebet einschließen".

Anschließend verlas Prof. S. Krawczyński die Laudatio, in der er unter anderem Folgendes betonte: „Die akademische Gemeinschaft der Hochschulen Krakaus - die Päpstliche Universität Johannes Paul II. und die Akademie für Musik - haben nun die Ehre, ihre große Dankbarkeit für die Annahme der höchsten akademischen Würde, den Ehrendoktortitel, auszudrücken. Wir danken dafür, dass Ihre Heiligkeit durch die Annahme dieser Auszeichnung $\mathrm{zu}$ einem Mitglied unserer akademischen Gemeinschaft werden und diese auf 
der theologischen, musikalischen und menschlichen Ebene bereichern". Es folgten H. H. Prof. W. Zyzak, Rektor der UPJPII, und Prof. Z. Łapiński, Rektor der Akademie für Musik in Krakau, die den Beschluss der jeweiligen Senate verlasen und dem Heiligen Vater Benedikt XVI. das Diplom des Doktor honoris causa beider Hochschulen überreichten.

Der emeritierte Papst Benedikt XVI. unterstrich in seiner Doktorrede besonders seine enge Beziehung zu Polen und zur Musik: „In diesem Augenblick kann ich lediglich meine aufrichtigste und herzlichste Dankbarkeit für die große Ehre ausdrücken, die Sie mir mit der Verleihung der Ehrendoktorwürde erwiesen haben. Ich danke dem Großkanzler, Seiner geschätzten Eminenz Kardinal Stanisław Dziwisz, und dem akademischen Rat der beiden Hochschulen. Vor allem freue ich mich darüber, dass sich auf diese Weise meine Verbundenheit mit Polen und Krakau, der Heimat unseres großen heiligen Johannes Paulus II. weiter vertieft hat. Denn ohne ihn wäre mein geistlicher und theologischer Weg schwerlich vorstellbar. Mit seinem lebendigen Beispiel zeigte er uns auch, wie sich die Freude an edler Sakralmusik und die Aufgabe der gemeinsamen Teilnahme an der Liturgie miteinander verbinden lassen, die Freude und die demütige Einfachheit, den Glauben zu zelebrieren... In dieser Stunde kann man wohl zu Recht die grundlegende Frage stellen: Was ist Musik eigentlich? Woher kommt sie und worauf zielt sie ab? Ich denke, man kann auf drei 'Orte' verweisen, denen die Musik entspringt. Ihre erste Quelle ist die Erfahrung der Liebe. Wann immer Menschen von der Liebe bezaubert waren, öffnete sich ihnen eine andere Dimension ihrer Existenz, eine neue Weite und Perspektive der Wirklichkeit. Sie erweckte auch neue Möglichkeiten sich selbst auf andere Art auszudrücken. Poesie, Gesang und Musik im Allgemeinen sind aus dieser beeindruckenden Erfahrung entstanden, aus dieser Öffnung auf einen neuartigen Lebensbereich hin. Die zweite Quelle der Musik ist die Erfahrung der Trauer, die Berührung mit Tod, Schmerz und den Abgründen des Lebens. Auch in diesem Fall eröffnen sich in entgegengesetzter Richtung neue Dimensionen der Realität, auf die man durch Worte allein keine Antworten finden kann. Der dritte Ort, dem die Musik entströmt, ist schließlich die Begegnung mit dem, was Göttlich, also von Beginn an auf irgendeine Weise so zu definieren, und dem, was menschlich ist. Hier ist am intensivsten etwas vollkommen Anderes und etwas vollkommen Großes zugegen, das im Menschen neue Ausdrucksweisen seiner selbst erweckt. Vielleicht kann man sagen, dass uns im Wesentlichen auch in diesen beiden verbleibenden Bereichen - in der Liebe und im Tod - das Geheimnis Gottes 


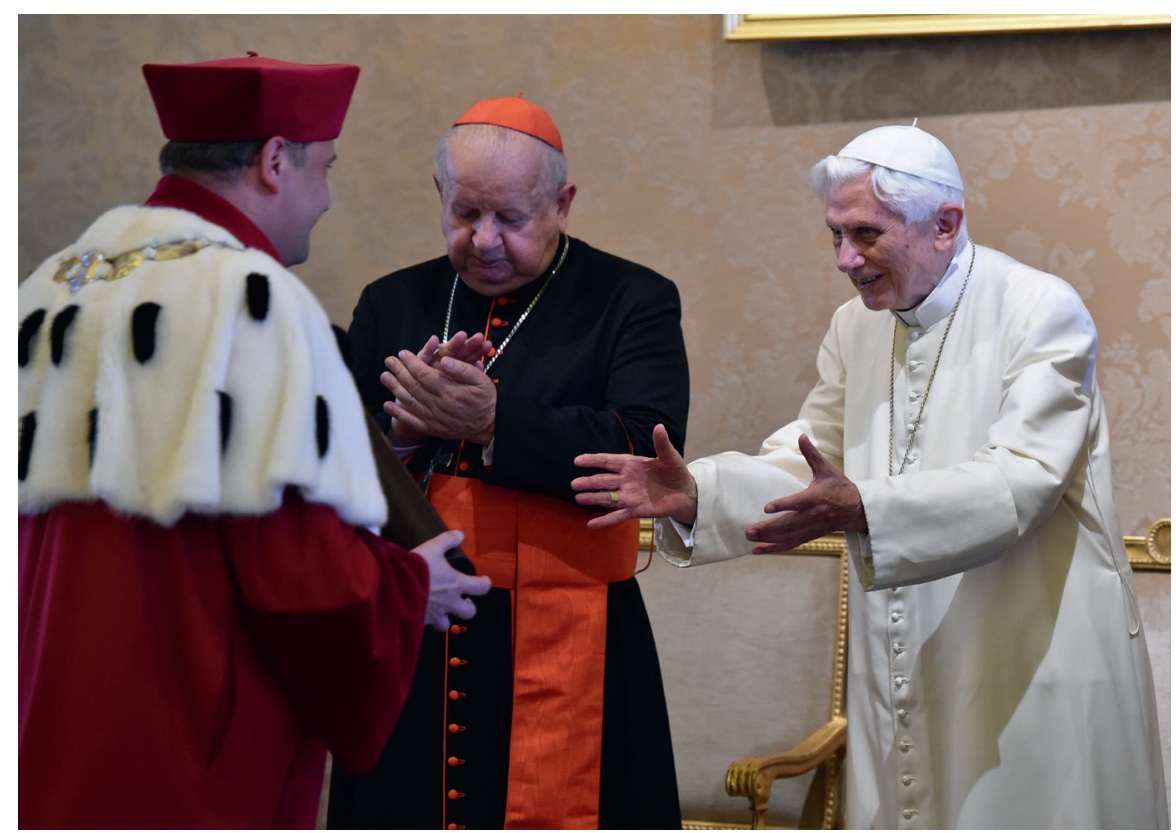

berührt und dass deshalb in diesem Sinn die Berührung durch Gott insgesamt die Quelle der Musik ist. Es ist ergreifend wahrzunehmen, dass den Menschen zum Beispiel in den Psalmen der Gesang allein nicht genügt, und wir dort die Berufung auf alle Instrumente finden: die verborgene Musik des Geschöpfes wird angeregt, seine geheimnisvolle Sprache. Mit dem Buch der Psalmen, in dem wir es mit den beiden Motiven der Liebe und des Todes zu tun haben, befinden wir uns unmittelbar an der Quelle der Musik der Kirche Gottes. Man kann sagen, dass die Qualität der Musik von der Reinheit und Vortrefflichkeit der Begegnung mit dem Göttlichen, von der Erfahrung der Liebe und des Leides abhängt. Je reiner und wahrhafter diese Erfahrung ist, desto reiner und vortrefflicher wird auch die Musik sein, die daraus erwächst und sich entfaltet... An dieser Stelle möchte ich einen Gedanken äußern, der mich in letzter Zeit sehr beschäftigt, ja immer mehr, seit die verschiedenen Kulturen und Religionen häufiger miteinander in Verbindung treten. Wie unterschiedlich die Kulturen und Religionen auch sein mögen, sind in ihren Umkreis doch stets große Literatur, prächtige Architektur, Malerei sowie herrliche Bildhauerei zugegen. Und überall ist auch Musik. In keinem anderen kulturellen Umfeld gibt es jedoch Musik, deren Größe mit der im Kontext des christlichen Glaubens entstandenen zu vergleichen ist: von Palestrina zu Bach und Händel, bis hin 
zu Mozart, Beethoven und Bruckner. Die abendländische Musik ist etwas Außergewöhnliches, in anderen Kulturen gibt es nichts Gleichwertiges. Das soll uns zum Nachdenken bringen... Das Wirken der beiden Hochschulen, die mir dieses Doktorat honoris causa verleihen, leistet einen wesentlichen Beitrag dazu, dass das große Geschenk der Musik, die der Tradition des christlichen Glaubens entspringt, lebendig bleibt und dabei hilft, dass die schöpferische Kraft des Glaubens auch in Zukunft nicht erlösche. Dafür danke ich Euch von Herzen, nicht nur für die Ehre, die Ihr mir erwiesen haben, sondern für die gesamte Arbeit, die Ihr im Dienst an der Schönheit des Glaubens leistet. Der Herr segne Euch alle!"

In Verbindung mit der dargestellten Feierlichkeit gaben Kardinal Stanisław Dziwisz, Metropolit von Krakau und Großkanzler der UPJPII, H. H. Prof. Dr. hab. Wojciech Zyzak, Rektor der UPJPII sowie Prof. Dr. hab. Zdzisław Łapiński, Rektor der Akademie für Musik in Krakau, ein spezielles Kommuniqué heraus. Mit der Verleihung der Ehrendoktorwürde an den emeritierten Heiligen Vater Benedikt XVI. bringen die beiden Hochschulen ihre Huldigung und Dankbarkeit gegenüber der Persönlichkeit des Papstes zum Ausdruck, gleichzeitig ist sie auch eine Auszeichnung für die Akademie für Musik in Krakau, für die Päpstliche Universität Johannes Paul II. in Krakau sowie für die gesamte Erzdiözese Krakau. 